PROCEEDINGS OF THE

AMERICAN MATHEMATICAL SOCIETY

Volume 135, Number 8, August 2007, Pages 2573-2579

S 0002-9939(07)08761-8

Article electronically published on March 22, 2007

\title{
A QUASICONFORMAL MAPPING CLASS GROUP ACTING TRIVIALLY ON THE ASYMPTOTIC TEICHMÜLLER SPACE
}

\author{
KATSUHIKO MATSUZAKI
}

(Communicated by Juha M. Heinonen)

\begin{abstract}
For an analytically infinite Riemann surface $R$, the quasiconformal mapping class group MCG $(R)$ always acts faithfully on the ordinary Teichmüller space $T(R)$. However in this paper, an example of $R$ is constructed for which $\operatorname{MCG}(R)$ acts trivially on its asymptotic Teichmüller space $A T(R)$.
\end{abstract}

\section{$\S 1$. InTRODUCTION AND STATEMENT OF RESUlts}

The asymptotic Teichmüller space $A T(R)$ of a Riemann surface $R$ is a certain quotient space of the Teichmüller space $T(R)$. It was first introduced in [7] when $R$ was the hyperbolic plane and in 3, 4 when $R$ was an arbitrary hyperbolic Riemann surface.

A quasiconformal homeomorphism $f$ of $R$ is called asymptotically conformal if, for every $\varepsilon>0$, there exists a compact subset $V$ of $R$ such that the maximal dilatation $K(f)$ of $f$ is less than $1+\varepsilon$ on $R-V$. Two quasiconformal homeomorphisms $f_{1}$ and $f_{2}$ of $R$ are said to be asymptotically equivalent if there exists an asymptotically conformal homeomorphism $h: f_{1}(R) \rightarrow f_{2}(R)$ such that $f_{2}^{-1} \circ h \circ f_{1}: R \rightarrow R$ is homotopic to the identity relative to the ideal boundary at infinity of $R$. The asymptotic Teichmüller space $A T(R)$ is the set of all asymptotic equivalence classes $[f]_{A}$ of quasiconformal homeomorphisms $f$ of $R$. Since a conformal homeomorphism is asymptotically conformal, the ordinary Teichmüller equivalence is stronger than the asymptotic equivalence. Hence there exists a natural projection $\pi: T(R) \rightarrow A T(R)$ that maps each Teichmüller equivalence class $[f] \in T(R)$ to the asymptotic equivalence class $[f]_{A} \in A T(R)$.

For the complex conjugate $R^{*}$ of $R$, let $B\left(R^{*}\right)$ be the complex Banach space of all bounded holomorphic quadratic differentials on $R^{*}$ with hyperbolic supremum norm and $B_{0}\left(R^{*}\right)$ the closed subspace of $B\left(R^{*}\right)$ consisting of all bounded holomorphic quadratic differentials $\varphi$ that vanish at infinity. Here we say that $\varphi$ vanishes at infinity if for every $\epsilon>0$ there exists a compact subset $V^{*} \subset R^{*}$ such that the norm of $\left.\varphi\right|_{R^{*}-V^{*}}$ taken off $V^{*}$ is less than $\epsilon$. It is proved in $[3$ that the asymptotic Teichmüller space $A T(R)$ is a complex Banach manifold modeled on the quotient Banach space $B\left(R^{*}\right) / B_{0}\left(R^{*}\right)$. In fact, $A T(R)$ is embedded in $B\left(R^{*}\right) / B_{0}\left(R^{*}\right)$ as a bounded contractible domain, as is proved in [5]. This is the unique complex

Received by the editors August 16, 2005 and, in revised form, April 19, 2006.

2000 Mathematics Subject Classification. Primary 30F60; Secondary 32G15.

Key words and phrases. Analytically infinite Riemann surface, quasiconformal mapping class group, asymptotic Teichmüller space.

(C)2007 American Mathematical Society Reverts to public domain 28 years from publication 
structure on $A T(R)$ such that the projection $\pi: T(R) \rightarrow A T(R)$ is holomorphic with respect to the complex structure on $T(R)$.

A pseudo-distance $d_{A}$ on $A T(R)$ is induced from the Teichmüller distance $d$ on $T(R)$ as the quotient: $d_{A}\left([f]_{A},[g]_{A}\right)=\inf d\left(\left[f^{\prime}\right],\left[g^{\prime}\right]\right)$ where the infimum is taken over all $\left[f^{\prime}\right] \in \pi^{-1}\left([f]_{A}\right)$ and all $\left[g^{\prime}\right] \in \pi^{-1}\left([g]_{A}\right)$. It is proved in [3] that the fiber $\pi^{-1}\left([f]_{A}\right)$ for each $[f]_{A} \in A T(R)$ is a closed submanifold of $T(R)$. Also the above infimum is coincident with the infimum taken over all $\left[g^{\prime}\right] \in \pi^{-1}\left([g]_{A}\right)$ with $\left[f^{\prime}\right] \in \pi^{-1}\left([f]_{A}\right)$ fixed arbitrarily. Hence $d_{A}$ turns out to be a complete distance which is called the asymptotic Teichmüller distance.

The asymptotic Teichmüller space $A T(R)$ is of interest only when $R$ is analytically infinite. Otherwise $A T(R)$ is trivial, that is, it consists of just one point. Conversely, if $R$ is analytically infinite, then $A T(R)$ is not trivial. This is because the Banach space $B_{0}\left(R^{*}\right)$ is a proper subspace of $B\left(R^{*}\right)$ whenever $R$ is analytically infinite. See the Corollary in Section 6 of [1]. In fact, $B_{0}\left(R^{*}\right)$ is a separable Banach space whereas $B\left(R^{*}\right)$ is not separable. Hence $B\left(R^{*}\right) / B_{0}\left(R^{*}\right)$ is not separable and in particular infinite dimensional.

The quasiconformal mapping class is a homotopy equivalence class $[g]$ of quasiconformal automorphisms $g$ of $R$, and the quasiconformal mapping class group $\operatorname{MCG}(R)$ is the group of all these mapping classes. Here the homotopy is also relative to the ideal boundary at infinity. Every element $[g] \in \operatorname{MCG}(R)$ induces a biholomorphic automorphism of $T(R)$ by $[f] \mapsto\left[f \circ g^{-1}\right]$, which is also isometric with respect to the Teichmüller distance $d$. In this way, $\operatorname{MCG}(R)$ acts on $T(R)$, and a representation $\iota: \operatorname{MCG}(R) \rightarrow \operatorname{Aut}(T(R))$ is given. Similarly, $[g] \in \operatorname{MCG}(R)$ induces a biholomorphic isometric automorphism of $A T(R)$ by $[f]_{A} \mapsto\left[f \circ g^{-1}\right]_{A}$. Thus we have a representation $\iota_{A}: \operatorname{MCG}(R) \rightarrow \operatorname{Aut}(A T(R))$.

It is proved in 2] that the representation $\iota: \operatorname{MCG}(R) \rightarrow \operatorname{Aut}(T(R))$ is injective (faithful) if $R$ is analytically infinite, or more precisely, unless $R$ is analytically finite of either $(0,3),(0,4),(1,1),(1,2)$ or $(2,0)$ type. In fact, $\iota$ is bijective (see 8$]$ ). On the other hand, it is also proved in $\left[2\right.$ that the representation $\iota_{A}: \operatorname{MCG}(R) \rightarrow$ $\operatorname{Aut}(A T(R))$ is injective precisely when $R$ is either the unit disk or a once-punctured disk. This fact is natural in some sense, for different actions of mapping classes of $R$ on $T(R)$ can shrink to the same action on $A T(R)$ under the projection $\pi$ : $T(R) \rightarrow A T(R)$. Concerning this matter, we will see in this paper that even the following extreme case can occur.

Theorem 1. There exists an analytically infinite Riemann surface $R^{\circ}$ such that the quasiconformal mapping class group $\mathrm{MCG}\left(R^{\circ}\right)$ acts trivially on the asymptotic Teichmüller space $A T\left(R^{\circ}\right)$, namely, the kernel of the homomorphism $\iota_{A}$ is entirely $\operatorname{MCG}\left(R^{\circ}\right)$.

\section{$\S 2$. Construction of the Riemann surface $R^{\circ}$}

In [9], we have obtained an analytically infinite Riemann surface $R$ such that the quasiconformal mapping class group $\operatorname{MCG}(R)$ consists only of a countable number of elements. This mapping class group also holds a property that every mapping class has an asymptotically conformal representative as an automorphism of the base surface $R$. However, this is weaker than the statement of Theorem 1 which is equivalent to saying that every mapping class has an asymptotically conformal representative as an automorphism of every surface $R^{\prime}$ quasiconformally equivalent 
to $R$. For this property, it suffices to prove that every mapping class has an eventually trivial representative as an automorphism of the base surface $R$. Here we say that a quasiconformal automorphism $f$ of $R$ is eventually trivial if there exists a topologically finite subsurface $V$ of $R$ such that $f$ is the identity on $R-V$. The Riemann surface $R^{\circ}$ as in Theorem 1 is constructed from $R$ by removing a countable number of points appropriately so that every quasiconformal mapping class should be eventually trivial.

A pair of pants $P$ is a hyperbolic surface with geodesic boundaries $c$ whose interior is homeomorphic to a three-punctured sphere. Every pair of pants admits a canonical orientation reversing isometric involution. The fixed point loci of this involution consist of three geodesic segments $\sigma$, which we call the symmetry axes. Cutting along the symmetry axes, we have two congruent right-angled hexagons $D$. Let $P_{0}$ be a pair of pants the lengths of whose geodesic boundary components are 0 ! and 1 ! and 1 !. Let $P_{1}$ be a pair of pants with the lengths 1 ! and 2 ! and 2 !. In the same way, for every non-negative integer $n$, let $P_{n}$ be a pair of pants with the lengths $n$ ! and $(n+1)$ ! and $(n+1)$ !. The three symmetry axes divide $P_{n}$ into two congruent right-angled hexagons $D_{n}$. The geodesic boundary components of length $n$ ! and $(n+1)$ ! in $P_{n}$ are denoted by $c_{n}$ and $c_{n+1}$ (right and left) respectively.

The Riemann surface $R$ is made of $2^{n+1}$ copies of $P_{n}$ for all $n \geq 0$ as follows. We take 2 copies of $P_{0}$ and glue the geodesic boundary component $c_{0}$ of each $P_{0}$ together. The resulting hyperbolic surface with 4 geodesic boundary components $c_{1}$ is denoted by $R_{1}$. Next take 4 copies of $P_{1}$ and glue the geodesic boundary component $c_{1}$ of each $P_{1}$ with the 4 boundary components of $R_{1}$. The resulting hyperbolic surface with 8 geodesic boundary components $c_{2}$ is denoted by $R_{2}$. Continuing this process, we obtain, for every $n \geq 1$, a hyperbolic surface $R_{n}$ with $2^{n+1}$ geodesic boundary components $c_{n}$ made of $R_{n-1}$ and $2^{n}$ copies of $P_{n-1}$. Then we take the exhaustion of these surfaces $R_{n}$, which is $R=\bigcup_{n=1}^{\infty} R_{n}$. Each connected component of $R-R_{n}$ is a neighborhood of an end, which is denoted by $E_{n}$. At each step of gluing, we give an appropriate amount of twist along the geodesic boundaries so that $R$ is a complete hyperbolic surface without ideal boundary at infinity.

To make $R^{\circ}$ from $R$, we puncture infinitely many pairs of pants $P_{n}$ in $R$. The removed point $q_{n}$ in $P_{n}$ is chosen at the middle of the symmetric axis connecting the right and left $c_{n+1}$ in $P_{n}$. In order to choose the punctured pairs of pants, we use a family of streams as is defined in the next two paragraphs. A pair of pants $P_{n}$ in $R$ is punctured if no stream in the family intersects it. Otherwise, it is not punctured. In this manner, we have a punctured Riemann surface $R^{\circ}$.

An $n$-stream $(n \geq 0)$ is a path starting from a pair of pants $P_{n}$ and going through $P_{n+1}, P_{n+2}, \ldots$ in order towards infinity. Each $n$-stream takes a right as its initial direction at $P_{n}$ and then continues to keep right until it reaches the turning point $P_{i}(i>n)$. The stream switches its direction from right to left once it is here at $P_{i}$. We call this integer $i$ the turning number for the stream. After $P_{i}$, it takes a left throughout.

We make a family of the streams satisfying the following conditions:

(1) Two 0-streams start from both the pairs of pants $P_{0}$.

(2) If a stream passes or starts from a pair of pants $P_{n}(n \geq 0)$, then, among the two adjacent pairs of pants $P_{n+1}$ to the $P_{n}$, the one where the stream 
does not pass has the two adjacent pairs of pants $P_{n+2}$ from which two $(n+2)$-streams start.

(3) Every pair of pants $P_{n}$ intersects at most one stream.

(4) All the turning numbers of the streams are mutually distinct.

Then we use this family to determine the punctured pairs of pants as explained above.

\section{$\S 3$. Geometry of the Riemann surface $R^{\circ}$}

The punctured Riemann surface $R^{\circ}$ has no ideal boundary at infinity. This can be seen from a general fact that if $R$ has no ideal boundary at infinity and $R^{\circ}$ is obtained by removing a totally disconnected closed set from $R$, then $R^{\circ}$ has no ideal boundary at infinity. Indeed, a Riemann surface has ideal boundary at infinity if and only if it has a simply connected subdomain $W$ such that the relative boundary $\partial W$ is connected and that the harmonic measure of $\partial W$ in $W$ is not full; if $R^{\circ}$ has this property then so does $R$. Remark that, since $R^{\circ}$ has no ideal boundary at infinity, homotopy in the definition of the Teichmüller equivalence for $T\left(R^{\circ}\right)$ can be regarded as free homotopy.

A boundary component of $P_{n} \cap R^{\circ}$ is not geodesic with respect to the intrinsic hyperbolic metric on $R^{\circ}$. For each of the three boundary components of $P_{n} \cap R^{\circ}$, there exists a unique simple closed geodesic in $R^{\circ}$ in its free homotopy class, which is denoted by $c_{n}^{\circ}$ or $c_{n+1}^{\circ}$. A subsurface of $R^{\circ}$ bounded by these three simple closed geodesics, $c_{n}^{\circ}, c_{n+1}^{\circ}$ right and $c_{n+1}^{\circ}$ left, is denoted by $P_{n}^{\circ}$, which is either a punctured or a non-punctured pair of pants. Similarly, $R_{n}^{\circ}$ is defined to be the subsurface of $R^{\circ}$ bounded by all $c_{n}^{\circ}$, and $E_{n}^{\circ}$ is defined to be a connected component of $R^{\circ}-R_{n}^{\circ}$.

The complete hyperbolic metric on $R^{\circ}$ is not less than the hyperbolic metric on $R$. This is because of a monotone property of the hyperbolic densities with respect to the inclusion relation between domains. This in particular implies that the length $\ell\left(c_{n}^{\circ}\right)$ of the geodesic $c_{n}^{\circ}$ in $R^{\circ}$ is not less than the length $\ell\left(c_{n}\right)$ of the geodesic $c_{n}$ freely homotopic to $c_{n}^{\circ}$ in $R$. On the other hand, for our particular surface $R^{\circ}, \ell\left(c_{n}^{\circ}\right)$ has the following upper bound in terms of $\ell\left(c_{n}\right)$.

Lemma 2. The length $\ell\left(c_{n}^{\circ}\right)$ is not greater than $4 \ell\left(c_{n}\right)=4 n$ !.

Proof. In the original surface $R$, consider an embedded annulus

$$
H_{\omega}\left(c_{n}\right)=\left\{p \in E_{n} \mid 0<d_{R}\left(c_{n}, p\right)<\omega\right\}
$$

called a half-collar for the simple closed geodesic $c_{n}$, where $d_{R}$ denotes the hyperbolic distance on $R$. By Proposition 5 and Lemma 3 in 9 , we see that the length of the shortest geodesic arc $\delta_{n}\left(\not \subset c_{n}\right)$ connecting $c_{n}$ to itself in $E_{n}$ is

$$
\ell\left(\delta_{n}\right)=2 \operatorname{arcsinh}\left\{\frac{\cosh ((n+1) ! / 2)}{\sinh (n ! / 4)}\right\}>n ! \times n .
$$

Hence uniform width $\omega$ of the half-collar $H_{\omega}\left(c_{n}\right)$ can be taken as

$$
\operatorname{arcsinh}\left\{\frac{\cosh ((n+1) ! / 2)}{\sinh (n ! / 4)}\right\}>\operatorname{arcsinh} 1=: \omega
$$

for every $n \geq 1$.

Let $A_{n}$ be the annular cover of $R$ with respect to the $c_{n}$. The half-collar $H_{\omega}\left(c_{n}\right)$ can be regarded as embedded in $A_{n}$. The value of the width $\omega=\operatorname{arcsinh} 1$ implies that their moduli are related as $m\left(H_{\omega}\left(c_{n}\right)\right)=m\left(A_{n}\right) / 4$. Since $\omega$ is less than 
$\ell\left(\delta_{n}\right) / 2$, the half-collar $H_{\omega}\left(c_{n}\right)$ does not contain any $q_{i}$ for $i \geq 0$. Hence $H_{\omega}\left(c_{n}\right)$ is also regarded as embedded in $R^{\circ}$ as well as in the annular cover $A_{n}^{\circ}$ of $R^{\circ}$ with respect to the $c_{n}^{\circ}$. Then their moduli satisfy an inequality $m\left(A_{n}^{\circ}\right) \geq m\left(H_{\omega}\left(c_{n}\right)\right)$. From the two inequalities above, we have $m\left(A_{n}^{\circ}\right) \geq m\left(A_{n}\right) / 4$. Since the geodesic length and the modulus of the annular cover are inversely proportional, this implies $\ell\left(c_{n}^{\circ}\right) \leq 4 \ell\left(c_{n}\right)$.

The measurement of $P_{n} \subset R$ given by Proposition 5 in [9] also yields the following estimate.

Proposition 3. Let $P_{n}$ be one of the pairs of pants in $R$ such that $P_{n} \cap R^{\circ}$ is punctured and let $c_{n}$ be the boundary component of $P_{n}$. Let $\eta_{n}$ be a closed or nonclosed curve in $P_{n} \cap R^{\circ}$. When $\eta_{n}$ is closed, assume that it is freely homotopic to $c_{n}$ in $R$ but not in $R^{\circ}$. When $\eta_{n}$ is not closed, assume that both end points of $\eta_{n}$ are on $c_{n}$, and that $\eta_{n}$ is not homotopic to a subarc of $c_{n}$ in $R^{\circ}$ fixing the end points. Then the hyperbolic length of $\eta_{n}$ in $R$ is greater than $n ! \times n$.

Proof. Consider the right-angled hexagon $D_{n}$, which is the symmetric half of $P_{n}$. Then the length of $\eta_{n}$ can be estimated by double the distance between the side of $D_{n}$ where $q_{n}$ lies and other sides not adjacent to it, which is either $\ell\left(c_{n+1}\right)$ or $\ell\left(\delta_{n}\right)$. Both are greater than $n ! \times n$.

This proposition gives the following estimate on the hyperbolic length of a closed geodesic on $R^{\circ}$, which corresponds to Lemma 3 in 9 .

Lemma 4. The length of a non-trivial closed geodesic $c^{\circ}\left(\neq c_{n}^{\circ}\right)$ in $R^{\circ}$ that intersects $E_{n}^{\circ}$ is greater than $n ! \times n$.

Proof. First assume that $c^{\circ}$ does not cross any $c_{i}$ trivially, which means that no subarcs of $c^{\circ}$ and $c_{i}$ together bound a simply connected domain. Let $m$ be the largest integer among $i$ for which $c^{\circ}$ has intersection with some $P_{i}$. Then the subarc $\eta_{m}=c^{\circ} \cap P_{m}$ satisfies the conditions as in Proposition 3. Since the length of $\eta_{m}$ measured by the hyperbolic metric on $R^{\circ}$ is not less than that on $R$, it is greater than $m ! \times m \geq n ! \times n$. If $c^{\circ}$ crosses some $c_{i}$ trivially, we replace the subarc of $c^{\circ}$ with the subarc of $c_{i}$, which together bounds a simply connected domain. By this replacement, the length measured in $R$ does not increase. Hence we may reduce our argument to the case under the first assumption.

\section{$\S 4$. Quasiconformal automorphisms of $R^{\circ}$}

In this section, we complete the proof of Theorem 1. First, we notice that the distribution of the punctures makes $R^{\circ}$ to satisfy the following property.

Lemma 5. Let $E_{n}$ and $E_{n}^{\prime}$ be any connected components of $R-R_{n}$ possibly coincident and let $h: E_{n} \rightarrow E_{n}^{\prime}$ be a conformal homeomorphism that is not the identity. Then the image of $E_{n} \cap R^{\circ}$ under $h$ is not coincident with $E_{n}^{\prime} \cap R^{\circ}$.

Proof. If the $P_{n} \subset \overline{E_{n}}$ and the $P_{n}^{\prime} \subset \overline{E_{n}^{\prime}}$ are of different type regarding puncture, there is nothing to prove. If $P_{n}$ and $P_{n}^{\prime}$ are both punctured, we consider one of pairs of $(n+1)$-streams starting from the adjacent $P_{n+1}$ and $P_{n+1}^{\prime}$. Since their turning numbers are distinct by construction, we have an index $i>n+1$ such that the corresponding $P_{i}$ and $P_{i}^{\prime}$ under $h$ are of different type. If neither $P_{n}$ nor $P_{n}^{\prime}$ are punctured, we consider the streams intersecting $P_{n}$ and $P_{n}^{\prime}$ respectively. Since 
their turning numbers are distinct, we again have an index $i>n$ such that $P_{i}$ and $P_{i}^{\prime}$ are of different type. Note that, in the above arguments, we mainly suppose the case where $h$ maps the right-hand side of $E_{n}$ to the right-hand side of $E_{n}^{\prime}$. Indeed the claim is rather easier to prove in the other case.

By this property of $R^{\circ}$ and a property of $R$ proved by Theorem 3 in $[9$, we have the following theorem concerning quasiconformal automorphisms of $R^{\circ}$, which is an essential ingredient for proving Theorem 1.

Theorem 6. Let $g^{\circ}: R^{\circ} \rightarrow R^{\circ}$ be an n/4-quasiconformal automorphism of the Riemann surface $R^{\circ}$ for any integer $n \geq 5$. Then, for each connected component $E_{n}^{\circ}$ of $R^{\circ}-R_{n}^{\circ}$, the restriction $\left.g^{\circ}\right|_{E_{n}^{\circ}}: E_{n}^{\circ} \rightarrow R^{\circ}$ is homotopic to the inclusion map $\left.i d\right|_{E_{n}^{\circ}}: E_{n}^{\circ} \rightarrow R^{\circ}$ in $R^{\circ}$.

Proof. Since a puncture is a removable singularity for a quasiconformal map, $g^{\circ}$ : $R^{\circ} \rightarrow R^{\circ}$ extends to a quasiconformal automorphism $g: R \rightarrow R$ of the original surface $R$. The maximal dilatation of $g$ is bounded by $n / 4 \leq n$. Then, by Theorem 3 and its proof in 9, the restriction $\left.g\right|_{E_{n}}: E_{n} \rightarrow R$ is homotopic to a conformal homeomorphism $h: E_{n} \rightarrow E_{n}^{\prime}$ onto a connected component $E_{n}^{\prime}$ of $R-R_{n}$.

Suppose to the contrary that $\left.g^{\circ}\right|_{E_{n}^{\circ}}$ is not homotopic to $\left.i d\right|_{E_{n}^{\circ}}$. There are two cases to be considered: $h \neq i d_{E_{n}}$ and $h=i d_{E_{n}}$. In the former case, the image of $E_{n} \cap R^{\circ}$ under $h$ is not coincident with $E_{n}^{\prime} \cap R^{\circ}$ by Lemma 5 . Hence in both cases, there exists some $c_{m}=\partial E_{m} \subset \overline{E_{n}}(m \geq n)$ such that $g\left(c_{m}\right)$ and $c_{m}^{\prime}:=h\left(c_{m}\right)$ are freely homotopic in $R$ but not in $R^{\circ}$. Then we see that either the geodesic $g\left(c_{m}\right)^{\circ}$ freely homotopic to $g\left(c_{m}\right)$ in $R^{\circ}$ has intersection with $h\left(E_{m}\right)^{\circ}$ or the geodesic $g^{-1}\left(c_{m}^{\prime}\right)^{\circ}$ has intersection with $E_{m}^{\circ}$. Without loss of generality, we may assume the former case. By Lemma 2, the geodesic length $\ell\left(c_{m}^{\circ}\right)$ in $R^{\circ}$ is not greater than $4 m$ !. By Lemma 4 , the geodesic length $\ell\left(g\left(c_{m}\right)^{\circ}\right)$ is greater than $m ! \times m$. Thus the maximal dilatation of $g$ is greater than $m / 4 \geq n / 4$. However, this is a contradiction.

Corollary 7. Every quasiconformal mapping class $[g] \in \operatorname{MCG}\left(R^{\circ}\right)$ has an eventually trivial representative $g_{0}: R^{\circ} \rightarrow R^{\circ}$.

Proof. For a $K$-quasiconformal automorphism $g$ of $R^{\circ}$, take an integer $n$ satisfying $n / 4>K$. Then, applying Theorem 6 to $g$, we see that $g$ is homotopic to the inclusion map on $R^{\circ}-R_{n}^{\circ}$. We modify $g$ to a quasiconformal automorphism $g_{0}$ of $R^{\circ}$ homotopic to $g$ such that $g_{0}$ is precisely the identity on $R^{\circ}-R_{n}^{\circ}$. Since $R_{n}^{\circ}$ is topologically finite, such a modification is always possible.

Proof of Theorem 1. Let $f: R^{\circ} \rightarrow S^{\circ}$ be any quasiconformal homeomorphism of the Riemann surface $R^{\circ}$ onto another Riemann surface $S^{\circ}$ and let $[g] \in \operatorname{MCG}\left(R^{\circ}\right)$ be any quasiconformal mapping class. By Corollary 7 , there is an eventually trivial representative $g_{0}$ in $[g]$. Then $f \circ g \circ f^{-1}$ is homotopic to $f \circ g_{0} \circ f^{-1}$. Here $f \circ g_{0} \circ f^{-1}$ is the identity on $S^{\circ}-f\left(R_{n}^{\circ}\right)$ for some $n$, and in particular it is an asymptotically conformal automorphism of $S^{\circ}$. This implies that the action of every $[g] \in \operatorname{MCG}\left(R^{\circ}\right)$ fixes each asymptotic equivalence class $[f]_{A} \in A T\left(R^{\circ}\right)$, namely, $\operatorname{MCG}\left(R^{\circ}\right)$ acts trivially on $A T\left(R^{\circ}\right)$.

Remark. Note that $R^{\circ}$ as well as $R$ satisfies the property that, for every $L>0$, the number of simple closed geodesics whose lengths are less than $L$ is finite. Actually, it is proved in [6] that if a Riemann surface does not satisfy this property, then the quasiconformal mapping class group has no common fixed point in the asymptotic 
Teichmüller space and in particular it acts non-trivially. Also Theorem 6 implies that $\operatorname{MCG}\left(R^{\circ}\right)$ consists of a countable number of elements, as does $\operatorname{MCG}(R)$. In general, we will prove in the forthcoming paper 10] that, if $\mathrm{MCG}(R)$ has a common fixed point in $A T(R)$, then $\mathrm{MCG}(R)$ must be countable.

\section{REFERENCES}

[1] C. Earle and F. Gardiner, Geometric isomorphisms between infinte dimensional Teichmüller spaces, Trans. Amer. Math. Soc. 348 (1996), 1163-1190. MR.1322950 (96h:32024)

[2] C. Earle, F. Gardiner and N. Lakic, Teichmüller spaces with asymptotic conformal equivalence, I.H.E.S. Preprint (1995).

[3] C. Earle, F. Gardiner and N. Lakic, Asymptotic Teichmüller space, Part I: the complex structure, In the tradition of Ahlfors and Bers, Contemp. Math., vol. 256, AMS, 2000, pp. 1738. MR $1759668(2001 \mathrm{~m}: 32029)$

[4] C. Earle, F. Gardiner and N. Lakic, Asymptotic Teichmüller space, Part II: the metric structure, In the tradition of Ahlfors and Bers III, Contemp. Math., vol. 355, AMS, 2004, pp. 187-219. MR.2145063 (2006g:30078)

[5] C. Earle, V. Markovic and D. Saric, Barycentric extension and the Bers embedding for asymptotic Teichmüller space, Complex manifolds and hyperbolic geometry, Contemp. Math., vol. 311, AMS, 2002, pp. 87-105. MR1940165 (2003i:30072)

[6] E. Fujikawa, The action of geometric automorphisms of asymptotic Teichmüller spaces, Michigan Math. J. 54 (2006), 269-282. MR2252759

[7] F. Gardiner and D. Sullivan, Symmetric structures on a closed curve, Amer. J. Math. 114 (1992), 683-736. MR1175689 (95h:30020)

[8] V. Markovic, Biholomorphic maps between Teichmüller spaces, Duke Math. J. 120 (2003), 405-431. MR2019982 (2004h:30058)

[9] K. Matsuzaki, A countable Teichmüller modular group, Trans. Amer. Math. Soc. 357 (2005), 3119-3131. MR2135738 (2006f:30052)

[10] K. Matsuzaki, Quasiconformal mapping class groups having common fixed points on the asymptotic Teichmüller spaces, J. Analyse Math., to appear.

Department of Mathematics, Ochanomizu University, Tokyo 112-8610, Japan

Current address: Department of Mathematics, Okayama University, Okayama 700-8530, Japan

E-mail address: matsuzak@math.okayama-u.ac.jp 